\title{
Loss of external rotation after open Bankart repair: an important prognostic factor for patient satisfaction
}

\author{
Hans Rahme • Ola Vikerfors • Lena Ludvigsson • \\ Maria Elvèn · Karl Michaëlsson
}

Received: 14 March 2009/Accepted: 29 October 2009/Published online: 28 November 2009

(C) Springer-Verlag 2009

\begin{abstract}
The repair of the capsuloligamentous complex during shoulder stabilisation procedures can be followed by a persistent restricted capacity of external rotation. The prognostic importance of this loss in external rotation for patient satisfaction has not previously been evaluated. We therefore followed 68 consecutive patients operated for recurrent traumatic unidirectional anterior instability of the glenohumeral joint to assess the association between loss of external rotation and patient satisfaction. All patients underwent open Bankart repair. Two independent observers carried out a follow-up (5 years on average) after surgery. At follow-up, recurrent dislocation had developed in four of the 68 patients $(6 \%)$. The median pre-operative Rowe score was 65 (range 42-87), which can be compared with 92 (range 46-100) at the follow-up. Three patients rated their outcome as poor, 13 as fair, 23 as good and 29 as excellent. There was a five-fold increased risk for a poor or fair outcome among patients with loss of external rotation in $0^{\circ}$ of abduction (age- and gender-adjusted odds ratio [OR] 5.3; 95\% confidence interval $[\mathrm{CI}] 1.3-22.0, P=0.0007)$. A linear association between the degree of loss in external rotation and patient dissatisfaction was found. The risk of being
\end{abstract}

H. Rahme · K. Michaëlsson

Department of Surgical Sciences, Section of Orthopaedics, University Hospital, Uppsala, Sweden

\section{H. Rahme ( $₫)$}

Elisabeth Hospital, Geijersgatan 20, 75226 Uppsala, Sweden e-mail: Hans.Rahme@aleris.se

O. Vikerfors · L. Ludvigsson · M. Elvèn

Department of Orthopaedics, Central Hospital, Västerås, Sweden

K. Michaëlsson

Uppsala Clinical Research Center, University Hospital,

Uppsala, Sweden dissatisfied, independent of recurrent dislocation, occasional pain, positive apprehension test, age and gender, more than doubled (OR 2.6; 95\% CI 1.4-4.8, $P=0.002$ ) for every $10^{\circ}$ of post-operative loss of external rotation. Loss of external rotation almost explained all of the variation in patient satisfaction with a population attributable risk of $0.85(95 \% \mathrm{CI}$ 0.20-0.94). We conclude that open Bankart repair with a modified Rowe procedure is an excellent surgical option regarding stability, but restriction in external rotation reduces the likelihood of a satisfied patient.

Keywords Shoulder instability · Bankart repair . Suture anchors $\cdot$ External rotation

\section{Introduction}

Traumatic recurrent unidirectional anterior shoulder dislocation is common in young people with a prevalence of $0.4 \%$ [13]. The average redislocation risk without surgery is $50 \%$, with an even more pronounced risk in patients under the age of 25 years $[6,7,17]$.

Although stabilizing surgery is an effective treatment for recurrent dislocations, recurrence of dislocations does occur. However, the risk of a redislocation after open Bankart repair is generally below $10 \%[11,14]$. Recurrence risk after arthroscopic surgery is approaching similar levels $[5,11,14]$. The optimal surgical procedure for anterior shoulder instability should restore stability without restriction of mobility. Repair of the capsuloligamentous complex during the shoulder stabilization procedures can, however, be followed by a persistent restricted capacity of external rotation, both after open $[9,12]$ and arthroscopic techniques $[10,16]$. The prognostic importance of this loss in external rotation for patient satisfaction has, to our knowledge, not 
previously been evaluated. Our hypothesis of this study was that loss of external rotation, not only recurrent dislocations, influences long-term patient satisfaction.

The aim of this study was therefore to ascertain the impact of loss of external rotation on patient satisfaction 5 years (on average) after surgery among patients who had undergone open Bankart repair according to Rowe's modified technique [18].

\section{Materials and methods}

Seventy-four consecutive patients were operated for traumatic unidirectional anterior shoulder instability. Before surgery, a modified Rowe score [18], the apprehension test at $90^{\circ}$ of abduction [4] and the degree of active outward rotation (with the arm at the side to reduce the impact of apprehension) were performed. All operations were done at the Department of Orthopaedics in Västerås, Sweden from 1989 to 1995 and all patients had pre- and post-operative radiographs taken of the affected shoulder. The cohort consisted of 59 men and 15 women with a mean initial age of 28 years (range 17-47). The dominant arm was involved in $42(57 \%)$ of the patients, where $41(55 \%)$ had sustained their initial dislocation during sports activities. All patients had at least two anterior dislocations and none had previous shoulder surgery. No patient showed any signs of osteoarthritis on pre-operative radiographs. Patients with large bony Bankart lesions requiring bone block operations were not included in the study. The modified Bankart operation was done under general anaesthesia with the patient in a supine position and the upper body elevated approximately $20^{\circ}$. A standard deltopectoral approach was used. At the beginning of the series, the coracoid process was osteotomised in 12 shoulders before the introduction of suture anchors. The subscapularis was divided into an L-shaped fashion and separated from the underlying capsule. If the rotator interval was widened, it was partially closed before opening of the capsule. Depending on the degree of capsular laxity, the capsule was then divided vertically 5-10 millimetres from the glenoid rim. In the 69 patients with a Bankart lesion, the anterior glenoid was decorticated and the lateral capsular flap sutured with two to four number 2 non-absorbable sutures through drill holes (9 patients), or with two to four Mitek GI $(n=32)$ or GII $(n=28)$ suture anchors (Mitek Surgical Products, Norwood, MA, USA) to the anterior glenoid rim with the arm in $30^{\circ}$ of abduction and $30^{\circ}$ of external rotation. Independent of technique used, we aimed at placing the anchors or drill holes adjacent to the bone-cartilage border. The medial flap was double-breasted over the lateral flap, which creates an anterior buttress as well as a tightening of the capsule. In the five shoulders with elongation of the anterior capsule but without a Bankart lesion the lateral capsular flap was sutured under the labrum and the medial flap double-breasted over the repair with the arm in $30^{\circ}$ degrees of abduction and $30^{\circ}$ of external rotation. The subscapularis tendon was resutured to its original attachment and the skin closed with interrupted sutures.

The patients were discharged from hospital on the first or second day post-surgery. Before discharge, standard antero-posterior and lateral radiographs were taken of the shoulder. A sling was worn from 1 to 7 days, depending on the choice of the patient. The patients were encouraged to use their arm in daily living but to avoid external rotation. After 3 weeks, progressive exercises were initiated in inward and outward rotation, abduction and forward flexion by gentle stretching. After 8 weeks, progressive resistive exercises were increased with the aid of a rubber band. At 4 months, full activity was allowed but the patients were told to hold the arm in front of their body when performing heavy athletic activities during the following 2 months. At the beginning of the series, radiographs were obtained at a 1-year follow-up but later excluded because all the anchors were found to be in unchanged position.

After an average of 60 months (range 27-108), a questionnaire on shoulder stability and pain was sent to all 74 patients. All but two responded (one patient had died and one could not be located). No information about a redislocation was observed when their hospital records were reviewed.

Of the 72 patients who answered the questionnaire, 68 underwent a follow-up performed by one of two independent observers (LL or ME). This follow-up occurred, on average, 63 months (range 27-110 months) after surgery. These attendees form our study base. At the clinical follow-up, the modified Rowe score [18], the Constant Murley score [3], the apprehension test in $90^{\circ}$ of abduction [4], the degree of active outward rotation (with the arm at the side to reduce the impact of apprehension) and the degree of patient satisfaction were recorded (poor, fair, good or excellent).

\section{Statistical analysis}

We estimated the influence of post-operative loss in external rotation on risk for an unsatisfactory (poor/fair) result. Logistic regression was used to estimate this risk as odds ratios (ORs) with 95\% confidence intervals (CIs), The procedure LOGISTIC by Statistical Analysis System (SAS 9.1, SAS Institute, Cary, NC, USA) was used for the computation. Overall patient satisfaction (excellent/good vs. fair/ poor) served as the dependent variable. In the multivariable model, we included as exposure the difference in external rotation before surgery and at follow-up as either a dichotomous variable (loss of external rotation vs no loss) or as a continuous variable (per $10^{\circ}$ of loss). Age (continuous), 
gender, occasional pain (yes vs. no), positive apprehension test (dichotomous) and the presence of a recurrent dislocation (dichotomous) served as covariates. We additionally evaluated if there existed a statistical interaction between loss of external rotation and preoperative external rotation by including a product interaction term of these variables in the multivariable model. The deviation from a non-linear association between loss of external rotation as a continuous variable and fair/poor outcome was tested by additional inclusion of a quadratic term of loss of external rotation in the model. In addition, we estimated ORs for a poor/fair result with redislocation after surgery.

The population attributable risk of a fair or poor result for loss of external rotation was calculated as

$$
\frac{p(\mathrm{OR}-1)}{[p(\mathrm{OR}-1)+1]}
$$

where $p$ is the prevalence of loss in external rotation among those with a poor/fair result. We additionally estimated the population attributable risk for those with either loss of external rotation or a redislocation after surgery. We used a paired (two-tailed) $t$ test to examine the difference in Rowe scores before surgery and at follow-up.

\section{Results}

The characteristics of the 68 patients are displayed in Table 1 . There were 14 women and 54 men included in the study. Their mean age at the time of surgery was 29 years (median age 27 years with a range of $17-47$ years). The average Rowe score was 88 at the latest follow-up (average 66 months) compared to 63 before surgery. The $95 \%$ CI for the difference in pre-operative and the latest follow-up Rowe score was $21-28(P<0.0001)$. The Constant-Murley score was not calculated pre-operatively but averaged 93 (range 48-100) at follow-up. During follow-up, four patients had experienced at least one recurrence. Of these four patients, one had a single redislocation during sleep after 5 years, while the other three had several recurrences during sport activities.

When patients judged their global shoulder function, 29 $(43 \%)$ graded that their shoulder function as excellent, 23
(34\%) believed it was good, $13(19 \%)$ deemed it fair and 3 (4\%) graded it as poor. The three poor results were combined with glenohumeral osteoarthritis in one patient, several recurrences in another patient and a post-operative deep infection in one patient. There were no reoperations performed during follow-up that was due to recurrent dislocation, but one patient underwent arthroscopy and manipulation under anaesthesia 4 months post-operatively because of stiffness.

With the arm at the side, the mean loss of active external rotation was $12^{\circ}\left(95 \%\right.$ CI $8-18^{\circ}$, range $\left.0-50^{\circ}\right)$. The mean external rotation was $66^{\circ}$ before surgery versus $54^{\circ}$ at the final follow-up.

Patients $(n=16)$ with loss of external rotation reported a five-fold increased risk of a less satisfactory (patient judgement of shoulder function as fair or poor, $n=16$ ) outcome (OR 5.3; 95\% CI 1.3-22.0, Table 2). When we controlled for recurrent dislocations, age, gender and a positive apprehension test in the multivariable model, the risk of a dissatisfied outcome was more than doubled (OR $2.5 ; 95 \%$ CI $1.5-4.3$ ) for every $10^{\circ}$ of post-operative loss of external rotation, (Table II). This association seemed to be linear. Loss in external rotation as a quadratic term in the multivariable model had a $P$ value of 0.38 . Inclusion of the variable occasional pain (yes vs. no) in the multivariate model (OR 2.6; 95\% CI 1.4-4.8) or exclusion of the three self-estimated poor patients from the analyses (OR 2.5; 95\% CI 1.4-4.4) changed the increased risk to a marginal degree. Furthermore, occasional pain per se was not significantly associated with a deficit in external rotation, recurrent dislocations or a positive apprehension test (all $P$ values $>0.2$ ). We found no interaction between loss of external rotation and preoperative rotation capacity $(P=0.85)$, i.e. the association between loss of external rotation and dissatisfaction was evident both among those with a good external rotation ability and among those with a more restricted external rotation before surgery.

The population attributable risk for a dissatisfied patient by a loss of external rotation was 0.85 (95\% CI $0.20-0.94$ ). Thus, loss of external rotation explained most of the variation in patient satisfaction.

A modest negative correlation was found between degree of loss in external rotation and the Rowe $(r=-0.38$,
Table 1 Characteristics of the patients

$A U$ arbitrary units

\begin{tabular}{llccc}
\hline Variable & Mean & SD & Median & Range \\
\hline Age (years) & 29 & 8 & 28 & $17-47$ \\
Pre-operative external rotation $\left(^{\circ}\right)$ & 66 & 12 & 60 & $30-80$ \\
Post-operative external rotation $\left(^{\circ}\right)$ & 54 & 18 & 60 & $30-80$ \\
Rowe score, pre-operative (AU) & 64 & 8 & 65 & $42-87$ \\
Rowe score, at one year (AU) & 93 & 7 & 95 & $65-100$ \\
Rowe score, after an average of 66 months (AU) & 88 & 12 & 92 & $46-100$ \\
\hline
\end{tabular}


Table 2 The association, estimated as odds ratios (ORs) with 95\% confidence intervals (CIs), between post-operative loss in external rotation and risk of a patient with a self-reported fair or poor outcome

\begin{tabular}{|c|c|c|c|c|c|c|c|c|}
\hline & $\begin{array}{l}\text { Number of } \\
\text { satisfied patients } \\
\text { (good/excellent) }\end{array}$ & $\begin{array}{l}\text { Number of } \\
\text { dissatisfied } \\
\text { patients } \\
\text { (fair/poor) }\end{array}$ & $\begin{array}{l}\text { Gender- and } \\
\text { age-adjusted } \\
\text { odds ratio }\end{array}$ & $95 \% \mathrm{CI}$ & $P$ value & $\begin{array}{l}\text { Multivariable } \\
\text { odds ratio }^{\mathrm{a}}\end{array}$ & $95 \% \mathrm{CI}$ & $P$ value \\
\hline No loss in external rotation & 28 & 3 & 1.0 & Reference & Reference & 1.0 & Reference & Reference \\
\hline Loss in external rotation & 24 & 13 & 5.3 & $1.2-22.0$ & 0.02 & 8.0 & $1.6-41.5$ & 0.01 \\
\hline Per $10^{\circ}$ loss in external rotation & NA & NA & 2.2 & $1.4-3.5$ & 0.0007 & 2.5 & $1.5-4.3$ & 0.0005 \\
\hline
\end{tabular}

${ }^{a}$ Adjusted for age (continuous), gender, positive apprehension test (yes/no) and relaxation (yes/no)

NA not applicable

$P=0.001)$ and Constant-Murley score $(r=-0.29$, $P=0.01)$. In addition, there was a moderate correlation at the end of follow-up between self-rated patient satisfaction and the Rowe score (gender- and age-adjusted Spearman rank correlation coefficient $r=0.65, P<0.0001)$ and the Constant-Murley score $(r=0.54, P<0.0001)$.

Fourteen patients $(21 \%)$ had a positive apprehension test. Of those with a positive apprehension test, four $(6 \%)$ had one or more redislocations after the Bankart operation. Expectedly, these four patients reported low satisfaction with the outcome of the operation. Compared with those without a redislocation, patients with a redislocation had a more than 10-fold increased risk of a fair or poor self-rated outcome (gender- and age-adjusted OR 12.0; 95\% CI 1.1128.4).

The OR for a fair or poor outcome with either loss of external rotation or a redislocation was 9.0 (95\% CI 1.640.3). The total population attributable risk for a dissatisfied patient by a loss of external rotation or a redislocation was 0.86 (95\% CI 0.34-0.97). Consequently, this risk was marginally higher than that for loss of external rotation alone.

\section{Discussion}

The key finding of the present study was the association between loss of external rotation after open Bankart repair and the substantial increased risk of patient dissatisfaction. With the low incidence of recurrent dislocations after surgery, loss of external rotation to a large extent explained long-term patient dissatisfaction. We are not aware of any earlier report examining this risk.

Several surgical procedures have been described for the treatment of recurrent anterior instability of the shoulder. The purpose of any surgical method for recurrent anterior instability is to restore stability without restriction of mobility. Bankart described repair of the detached labrum by suture of the capsule to the anterior glenoid rim [1], although more recent cadaver studies have emphasised that the labrum tear alone is not a sufficient cause of anterior shoulder instability [15]. In 1978, Rowe presented excellent long-term results with a recurrence rate of only $3.5 \%$ with his modification of the Bankart procedure [19]. We used Rowe's modification, although with suture anchors in the majority of our patients instead of the initial technique with drill holes through the anterior glenoid rim. The low rate of recurrences following the open Bankart procedure in our series is comparable to that reported by Rowe [19]. There are, however, reports with long-term results after open surgery with substantially higher frequency of recurrences [2, 20].

In our hands, the obvious drawback of this method is the restriction in external rotation that was frequently observed, which resulted in the patients' discontent. It is possible that we closed the rotator interval to medial in some patients and that the double breasting of the capsule was too extensive. Nevertheless, with the modified Bankart procedure, the extent of loss of external rotation in our series is of similar magnitude to that reported in several studies, with an estimated average loss of $13^{\circ}$ [21].

A major strength of the present study is that independent observers performed the follow-up examinations. The observers had no involvement whatsoever in the patients' surgical treatment or rehabilitation. In addition, our followup rate of $92 \%$ for the clinical examination is acceptable. Striking findings in this study were that $50 \%$ of the patients reported slight occasional pain in their operated shoulder several years after surgery and that $31 \%$ regarded their shoulder motion as being restricted despite that the average Constant and Rowe scores were 93 and 88, respectively. These discrepancies in patient satisfaction and functional outcome indicate that the scores might not convey and discover moderate impairments.

Our study has severable conceivable limitations. We cannot by our design identify the mechanisms that might explain the association between loss of external rotation and patient satisfaction. One can argue that the restricted external rotation in our patients was due to osteoarthritis. No radiographs were obtained at the final follow-up in this 
study which is a limitation. On the other hand, it has not been possible to disclose any correlation between patient satisfaction and radiological osteoarthritis using mediumand long-term follow-ups [8]. Furthermore, the degree of loss in external rotation 2 years post-surgery does not influence the degree of arthropathy after 15 years [8]. A further conceivable limitation is the different lengths of follow-up. Nevertheless, the differences in length of follow-up seemed to only marginally influence our estimates. Including time to follow-up in months as a covariate in our analyses changed our age- and gender-adjusted odds ratio for a fair or poor outcome to 2.37 (95\% CI 1.44-3.90) per $10^{\circ}$ loss of external rotation.

\section{Conclusion}

The main challenge with a Bankart repair is to balance between stability and mobility. Previous research has focused on stability, but we suggest that a retained external rotation is of major importance for mid-term patient satisfaction. We conclude that with the low recurrence rate after an open Bankart procedure, loss of external rotation is the main attributable predictor for patient dissatisfaction.

\section{References}

1. Bankart ASD (1938) The pathology and treatment of recurrent dislocation of the shoulder joint. Br J Surg 26:23-29

2. Berendes TD, Wolterbeek R, Pilot P, Verburg H, te Slaa RL (2007) The open modified Bankart procedure: outcome at followup of 10 to 15 years. J Bone Joint Surg Br 89:1064-1068

3. Constant CR, Murley AH (1987) A clinical method of functional assessment of the shoulder. Clin Orthop Relat Res 214:160-164

4. Hawkins RJB DJ (1985) Clinical evaluation of shoulder problems. In: Rockwood CAM FA (ed) The shoulder. Churchill Livingstone, Edinburgh, pp 1169-1170

5. Hobby J, Griffin D, Dunbar M, Boileau P (2007) Is arthroscopic surgery for stabilisation of chronic shoulder instability as effective as open surgery? a systematic review and meta-analysis of 62 studies including 3044 arthroscopic operations. J Bone Joint Surg Br 89:1188-1196
6. Hovelius L (1999) The natural history of primary anterior dislocation of the shoulder in the young. J Orthop Sci 4:307-317

7. Hovelius L, Augustini BG, Fredin H, Johansson O, Norlin R, Thorling J (1996) Primary anterior dislocation of the shoulder in young patients. A ten-year prospective study. J Bone Joint Surg Am 78:1677-1684

8. Hovelius L, Sandstrom B, Saebo M (2006) One hundred eighteen Bristow-Latarjet repairs for recurrent anterior dislocation of the shoulder prospectively followed for fifteen years: study II-the evolution of dislocation arthropathy. J Shoulder Elbow Surg 15:279-289

9. Hubbell JD, Ahmad S, Bezenoff LS, Fond J, Pettrone FA (2004) Comparison of shoulder stabilization using arthroscopic transglenoid sutures versus open capsulolabral repairs: a 5-year minimum follow-up. Am J Sports Med 32:650-654

10. Kartus C, Kartus J, Matis N, Forstner R, Resch H (2007) Longterm independent evaluation after arthroscopic extra-articular Bankart repair with absorbable tacks. A clinical and radiographic study with a seven to ten-year follow-up. J Bone Joint Surg Am 89:1442-1448

11. Lenters TR, Franta AK, Wolf FM, Leopold SS, Matsen FA (2007) Arthroscopic compared with open repairs for recurrent anterior shoulder instability. A systematic review and metaanalysis of the literature. J Bone Joint Surg Am 89:244-254

12. Magnusson L, Ejerhed L, Rostgard L, Sernert N, Kartus J (2006) Absorbable implants for open shoulder stabilization. A 7-8-year clinical and radiographic follow-up. Knee Surg Sports Traumatol Arthrosc 14:182-188

13. Milgrom C, Mann G, Finestone A (1998) A prevalence study of recurrent shoulder dislocations in young adults. J Shoulder Elbow Surg 7:621-624

14. Mohtadi NG, Bitar IJ, Sasyniuk TM, Hollinshead RM, Harper WP (2005) Arthroscopic versus open repair for traumatic anterior shoulder instability: a meta-analysis. Arthroscopy 21:652-658

15. Pouliart N, Marmor S, Gagey O (2006) Simulated capsulolabral lesion in cadavers: dislocation does not result from a bankart lesion only. Arthroscopy 22:748-754

16. Rhee YG, Ha JH, Cho NS (2006) Anterior shoulder stabilization in collision athletes: arthroscopic versus open Bankart repair. Am J Sports Med 34:979-985

17. Robinson CM, Dobson RJ (2004) Anterior instability of the shoulder after trauma. J Bone Joint Surg Br 86:469-479

18. Rowe CR (1988) The shoulder. Churchill Livingstone, New York

19. Rowe CR, Patel D, Southmayd WW (1978) The Bankart procedure: a long-term end-result study. J Bone Joint Surg Am 60:1-16

20. Salomonsson B, Abbaszadegan H, Revay S, Lillkrona U (2009) The Bankart repair versus the Putti-Platt procedure. Acta Orthop. doi:10.3109/17453670902988345

21. Warren RFC EV, Altchek DW (1999) The unstable shoulder. Lippincott-Raven, New York 